\title{
Expanding Family Medicine Scholarship to All Faculty: The Minnesota Model for Harmonizing Clinical Care, Education, and Research Missions
}

\author{
Jerica M. Berge, PhD, MPH, LMFT, CFLE, Charles Peek, PhD, \\ James T. Pacala, MD, MS, Patricia Adam, MD, Shailendra Prasad, MD, MPH, \\ Deborab Finstead, MS, Denise Windenburg, MHA, Jill Bengtson, MS, \\ Katie A. Loth, PhD, MPH, RD, Michele L. Allen, MD, MS, and \\ Angela Buffington, $P h D, M A, L P, A B P P-C N$
}

Background: The Department of Family Medicine and Community Health at the University of Minnesota engaged in a 5-year transformation to expand research and scholarship opportunities to all faculty. A harmonization framework was used to integrate the 3 missions of clinical care, education, and research to ensure that research and scholarship were an ongoing focus of the department.

Methods: The key elements of our transformation included as follows: (1) a general culture of inquiry, (2) harmonized leadership, (3) training and mentoring, and (4) infrastructure and resources. Components of each of these elements were intentionally instituted simultaneously and iteratively across the 5 years to provide robust and sustainable research and scholarship opportunities for all faculty.

Results: Outputs and outcomes of the harmonized transformation indicated that clinical and research faculty publications increased, and the percentage of clinical faculty trained in research and scholarship skills increased across the 5 years.

Conclusions: Important lessons learned during the harmonized transformation included the following: (1) key elements of the transformation need to be balanced as an ensemble, (2) cultural and organizational shifts take concerted effort and time, (3) embrace iteration: allow "bumps in the road" to propel the work forward, (4) transformation is financially feasible, (5) career research faculty can mutually benefit from clinical faculty engaging in scholarship, and (6) honor skepticism or disinterest and let people cultivate enthusiasm for research and scholarship rather than being forced. (J Am Board Fam Med 2021;34:1055-1065.)

Keywords: Faculty Development, Family Medicine, Fellowships and Scholarships, Leadership, Research Facilitators, Scholarly Communication

\section{Introduction}

Over the last several decades, there have been institutional- and national-level calls for increasing family

From the University of Minnesota, Department of Family Medicine and Community Health, Minneapolis, MN (JMB, CP, JTP, PA, SP, DF, DW, JB, KAL, MLA, AB).

Contributors Statement: JMB is the Vice Chair for Research in the department and conceptualized the paper, carried out the model described in the paper, assisted with data analysis and interpretation, wrote all drafts of the paper, and agrees to be accountable for all aspects of the work regarding the accuracy or integrity of any part of the work. $\mathrm{CP}$ assisted with the conceptualization of the paper and the data analysis. He also critically reviewed the paper, gave final approval of this version to be published, and agrees to be accountable for all aspects of the work regarding the accuracy or integrity of any part of the work. JTP is the Department Chair and conducted the data analysis. He also critically reviewed the paper, gave final approval of this version to be published, and agrees to be accountable for all medicine faculty engagement in research and scholarship efforts, including traditional research studies, publications, and presentations. ${ }^{1-6}$ An expanded research

aspects of the work regarding the accuracy or integrity of any part of the work. PA assisted with data acquisition and interpretation of the data. She also critically reviewed the paper, gave final approval of this version to be published, and agrees to be accountable for all aspects of the work regarding the accuracy or integrity of any part of the work. SP is the Vice Chair for Education in the department and assisted with conceptualizing the paper. He also critically reviewed the paper, gave final approval of this version to be published and agrees to be accountable for all aspects of the work regarding the accuracy or integrity of any part of the work. DF conducted the data analysis. She also critically reviewed the paper, gave final approval of this version to be published, and agrees to be accountable for all aspects of the work regarding the accuracy or integrity of any part of the work. DW is the Administrative Director of the Research Division in the department and assisted in conceptualizing the paper, 
and scholarship workforce is needed to guide improvement in care, operations, and implementation in the field of family medicine. ${ }^{6-11}$

While many faculty in departments of family medicine are interested in engaging in research and scholarship opportunities, numerous barriers prohibit or limit faculty engagement to the degree necessary for producing high-quality research and scholarship. Barriers often include time constraints due to clinical and administrative responsibilities, limited available resources to engage in research and scholarship, lack of specific writing or analytic skills, and lack of a supportive infrastructure to facilitate research and scholarship. ${ }^{12,13}$ In addition, academia can be experienced as disjointed between clinical, educational, and research missions, and this lack of harmonization may result in reduced opportunities to collaborate on research and scholarship. ${ }^{6,14}$

Numerous family medicine departments throughout the United States have attempted to reduce these known barriers through a variety of efforts: (1) expansion of institutional research and scholarship infrastructure, ${ }^{11}$ (2) increased access to networking and funding opportunities, ${ }^{15}$ (3) creation of peer support ${ }^{16}$ and writing accountability groups, ${ }^{17}$ (4) development and implementation of instructional programming to teach writing and analytic skills, ${ }^{18-20}$ and (5) providing structures and processes that ensure ongoing research and scholarship. ${ }^{21}$ While these past efforts have contributed important knowledge and set the stage for increasing research and scholarship efforts in family medicine at departmental and national levels, more work is needed to make these efforts sustainable. For example, "harmonizing" efforts across clinic, education, and research to attain integration across these missions will help to ensure that research and scholarship is an ongoing focus (ie, "part of the fabric of

carrying out the model, and contributed to the design of the study. She also critically reviewed the paper, gave final approval of this version to be published, and agrees to be accountable for all aspects of the work regarding the accuracy or integrity of any part of the work. JB assisted in carrying out the model and conducting the data analysis. She also critically reviewed the paper, gave final approval of this version to be published, and agrees to be accountable for all aspects of the work regarding the accuracy or integrity of any part of the work. KAL assisted in conceptualizing the paper and contributed to the design of the study. She also critically reviewed the paper, gave final approval of this version to be published, and agrees to be accountable for all aspects of the work regarding the accuracy or integrity of any part of the work. MLA assisted in conceptualizing the paper and contributed to the design of the study. She also critically reviewed the paper, gave final approval of this family medicine"), rather than a short-lived project or rotation. In addition, it is important to do this work simultaneously. For example, when providing more training and mentoring, barriers such as more time to engage in research and scholarship and infrastructure/ resources must also be addressed, in addition to a culture of research and scholarship being cultivated simultaneously, or the change will not be sustainable.

"Harmonization" is a framework identified by scholars to describe the importance of carrying out simultaneous goals across the missions of research, clinical care, and education in family medicine departments to create a culture of curiosity that ultimately improves health and health care. ${ }^{6,14}$ Peek et al explained what harmonization would look and feel like in this way, "Harmonization would be apparent to a patient or visitor walking into a clinic. For their care, they may be in contact with senior faculty and clinicians in training, some of whom are doing research. Their clinicians would be particularly adept at critical thinking and the research evidence regarding issues of concern to their care. They would see research study or curriculum development posters on the wallalong with quality improvement projects or metric boards. They would notice that this research is all about questions of importance to the actual practice of primary care. They might be asked if they want to participate in a study - which might be about care or different teaching methods. If so, they may encounter a research coordinator. They would ultimately feel like they are walking through an up-to-date clinic that is both creating and responding to new knowledgeand teaching new clinicians to use it well. ..."22

Understanding the importance of using a framework for harmonization ${ }^{6,14}$ across clinical care, education, and research missions to address the barriers to engaging in research and scholarship, the Department of Family Medicine and Community Health (DFMCH) at the University of Minnesota (UMN) worked diligently over

version to be published, and agrees to be accountable for all aspects of the work regarding the accuracy or integrity of any part of the work. AB assisted in carrying out the model, conceptualizing the paper, and contributing to the study's design. She also critically reviewed the paper, gave final approval of this version to be published and agrees to be accountable for all aspects of the work regarding the accuracy or integrity of any part of the work.

Funding: None.

Conflict of interest: There are no conflicting or competing interests to report.

Corresponding author: Jerica M. Berge, PhD, MPH,

LMFT, CFLE, 717 Delaware St. SE Room 425 Minneapolis, MN 55414, Telephone: 612-626-3693 (E-mail: jberge@umn.edu). 
5 years to develop a model that integrates our clinical, educational, and research missions within the department. This model represents a departmental transformation that builds on what is known about barriers to research and scholarship and extends the work of others by creating, implementing, and evaluating a comprehensive research infrastructure explicitly designed to increase research and scholarly output. Importantly, elements of these new processes were implemented simultaneously and iteratively to increase the likelihood of sustainability. The main aims of this article are as follows: (1) describe the transformation of the DFMCH in becoming a harmonized department that simultaneously supports a mission of research and scholarship alongside our clinic and educational missions; (2) identify the key elements, or "how-to," required to create a harmonized model to promote all-faculty research and scholarship; (3) report initial outputs and outcomes of the DFMCH's model to date; and (4) offer lessons learned for other medical school departments that may be interested in a similar transformation.

\section{Department of Family Medicine and Community Health (DFMCH) at the University of Minnesota} The DFMCH is part of the UMN Medical School. The Department has 98 faculty and multiple programs or centers with specialized research and clinical foci (ie, Sports Medicine, Healthy Eating and Activity Across the Lifespan (HEAL) Center; Program in Health Disparities Research, Program in Human Sexuality; Hospice and Palliative Care) and 8 residency programs accredited by the Accreditation Council for Graduate Medical Education (ACGME). Five of the 8 residency programs are located in urban areas, and 3 in rural Minnesota. The faculty makeup includes clinically oriented faculty, faculty educators, and career research faculty (ie, National Institutes of Healthfunded faculty).

\section{DFMCH Harmonization Transformation}

Approximately 5 years ago, the DFMCH used a harmonizing ${ }^{6,14}$ approach to integrate our research and scholarship mission alongside our other departmental missions of clinic and education. Even when academic family medicine programs are becoming more clinically focused on ensuring revenue generation, the DFMCH leadership valued the importance of the 3 missions mutually informing each other, ultimately improving health and healthcare. $^{23,24}$ Other reasons for our transformation motivation included several faculty within the department who were leading the harmonization efforts in the field of family medicine and the Dean of the medical school emphasized the importance of 1 publication per year for each faculty.

We defined "harmonized" as: (1) no 1 mission subordinates others; (2) each mission informs and strengthens the others (in real-time); (3) changes in 1 mission are translated into changes in the others; and (4) faculty experience the work of harmonized missions as 1 coherent job, not as competing "masters" and derive greater satisfaction and joy in practice. This intentional harmonization of the 3 missions included 4 key elements that were conceptualized simultaneously and evolved to bring about an integrated and sustainable approach to research and scholarship efforts: (1) promoting a general culture of inquiry, (2) harmonized leadership, (3) training and mentoring, and (4) infrastructure and resources (see Figure 1). We also intentionally used the words "research" and

\section{Figure 1. Key Elements of the DFMCH Harmonized Transformation.}

\section{DFMCH Harmonized Transformation Elements}

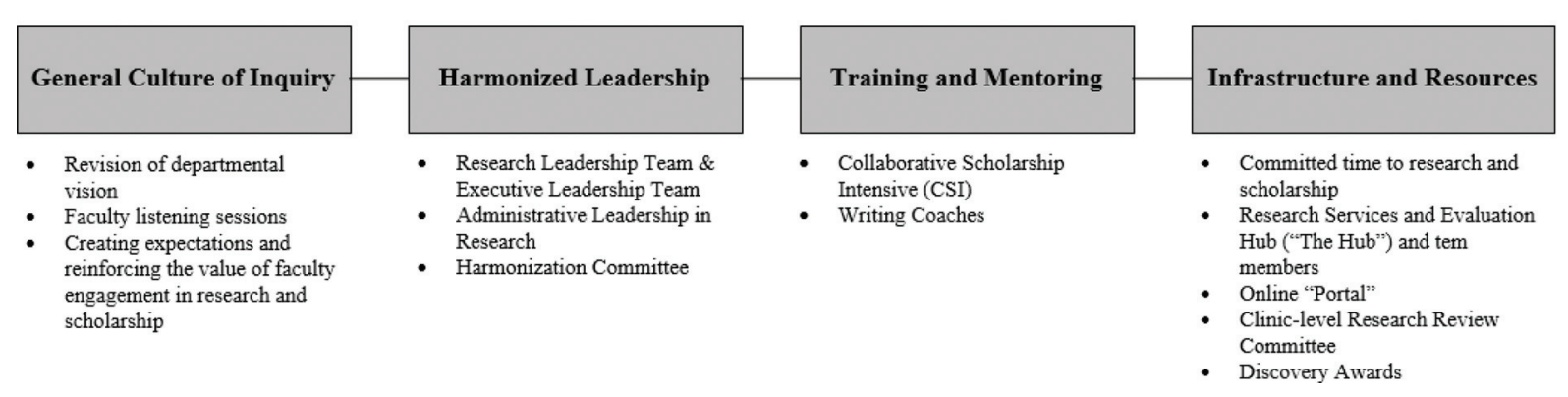


"scholarship" interchangeably to help faculty feel like engaging in the production of knowledge was accessible to all faculty. ${ }^{25}$

\section{General Culture of Inquiry}

A critical element of our harmonized transformation was facilitating a general culture of inquiry to promote faculty excitement and interest in engaging in research and scholarship activities.

\section{Revision of Departmental Vision}

One of the key activities that started the DFMCH harmonized transformation was revising our departmental vision statement. Our vision was amended to "We harmonize practice, education, and research through emerging models of care to improve individual, family, and community health." In addition, the definition of harmonization (presented above) was solidified and communicated to all faculty. This new vision set the stage for all our harmonized efforts.

\section{Faculty Listening Sessions}

To increase awareness of the benefits of research and scholarship, 2 steps were undertaken. First, program directors at the residency programs were surveyed about each program's needs around research and scholarship support and production. Second, the information gathered from these surveys was used to conduct structured faculty listening sessions. Faculty at each residency program were engaged in conversations through which they shared input about perceived barriers to scholarly output and support needed. As program directors, clinical faculty, and residents were able to discuss barriers, voice their needs, and then see their suggestions for increasing research and scholarship activities were implemented at the department level, there was more enthusiasm, willingness, and buy-in to engage in research and scholarship. In addition, a broader vision of research and scholarship was cultivated such that all types of discovery were valued (eg, quality improvement, evaluation, implementation) rather than just "traditional" research.

Creating Expectations and Reinforcing the Value of Faculty Engagement in Research and Scholarship

At the department level, there were leadership decisions to reinforce the culture for research and scholarship, such as building research and scholarship expectations into all faculty reviews and new faculty offer letters and holding residency and fellowship program directors responsible for reasonable research and scholarship production among their faculty. Department publication and grant funding goals were also set. These goals included as follows: (1) all clinical faculty should aim to publish 1 peer-reviewed article per year, (2) career research faculty should aim to publish 3 peer-reviewed articles per year, and (3) our department should strive to be in the top 3 of Blue Ridge rankings for most NIH-funded family medicine departments in the nation. In addition, when recruitment for new faculty hires occurred, faculty interested in engaging in scholarship and research efforts were prioritized to reinforce our harmonization model and focus on all faculty research and scholarship.

\section{Harmonized Leadership}

Over the 5-year transformation, key leadership shifts occurred to align the 3 missions of clinical care, education, and research.

\section{Harmonization Committee}

A committee was formed to ensure that our departmental commitment to harmonizing the 3 departmental missions of clinic, education, and research was realized. This committee met monthly and set the departmental transformation pace by working on projects that included components of clinic, education, and research and creating new departmental guidelines and recommendations to harmonize these efforts, with each mission reinforcing the other missions.

\section{Research Leadership Team and Executive Leadership Team}

A Research Leadership Team was created to facilitate the department's focus on research and scholarship. While this team was successful in moving the research efforts front-and-center in the department, it was determined that to harmonize our efforts across research, clinical care, and education, the Research Leadership team should evolve into an Executive Leadership Team to further reinforce our harmonized effort; thus the department chair (third author) appointed or conducted a national search (ie, Vice Chair for Research) for Vice Chairs for each of the 3 missions of Research (first author), Clinical Care (fourth author), and Education (fifth author). The 3 Vice Chairs continued building and expanding the 
harmonization efforts in the department. The Vice Chair for Research also created a research division vision of $\mathrm{I},{ }^{4}$ Inclusion, Integration, Innovation, and Identity, to align with and expand the DFMCH's harmonization efforts.

\section{Administrative Leadership in Research}

While the department already had an individual serving as the Administrative Research Director (seventh author), the position had initially been created to serve the career research faculty. The job responsibilities were adjusted and right sized to focus on coordinating research and scholarship efforts at a central level to ensure that harmonization was effectively and efficiently executed at the clinical and education levels and to serve as a dyadic partner with the Vice Chair for Research in harmonization efforts. In addition, as the research and scholarship efforts increased in the department, it was important to support these needs; thus, a research manager (eighth author) was hired to specifically oversee/supervise the research facilitators (described below) to ensure continued robust growth of research projects being submitted and publications being written by clinical faculty.

\section{Training and Mentoring}

Another crucial element of the harmonization transformation was providing training opportunities and mentoring focused on research and scholarship to increase clinical faculty's knowledge and skills.

\section{Collaborative Scholarship Intensive (CSI)}

A 6-month, 6-session faculty development program, called CSI, was created to increase research output among faculty with modest to no publications before enrolling. During CSI, participants are provided intensive instruction around phases of producing scholarly work, including project design, scholarly writing, project dissemination, and manuscript review. A key component of the course is structured work time, which allows protected writing time during which career research faculty partner 1-to-1 with participants to guide them through all phases of producing scholarly work. Five cohorts have completed CSI to date and have had significant before and after increases in their scholarly output (see Figure 2). ${ }^{20}$

\section{Writing Coaches}

Senior faculty and career research faculty were designated to meet 1-to-1 with faculty and guide them in manuscript completion. Initially appointed to help finish incomplete manuscripts, this role quickly evolved with the realization that faculty needed more intensive instruction in research methodology.

\section{Infrastructure and Resources}

A final essential element of our harmonized transformation was creating resources and infrastructure to enable faculty to engage in research and scholarship activities long-term successfully.

\section{Committed Time to Research and Scholarship}

To decrease a major barrier to faculty research and scholarship efforts- that being lack of time-clinical faculty were provided with $10 \%$ effort to engage in research and scholarship activities. This was structured to fit within an 80/20 faculty appointment, with 4 days in the clinic, 1 half-day allocated for administrative duties, and 1 half-day to engage in research and scholarship activities. In addition, the $80 \%$ included flexibility to engage in teaching, precepting, and other educational activities to ensure that harmonization across all 3 missions would be realized for every faculty.

\section{Research Services and Evaluation Hub ("the} Hub") and Team Members

A central location was established in the DFMCH called the Research Services and Evaluation Hub, or "The Hub," to provide research and scholarship resources and consultation services in real-time to department faculty. The Hub includes research facilitators, a statistician, research manager, database manager, data entry/survey development staff, computer programmer, 2 faculty experts in qualitative and quantitative methods, the Research Administrative Director, and the Vice Chair for Research.

1. Research facilitators and research staff. To ensure faculty have consistent access to expertise and resources to carry out research and scholarship, 1 full-time research facilitator was appointed to each of our $4 \mathrm{UMN}$ residency clinics to allow for stability and relationship formation with faculty to promote a collaborative, team atmosphere. Research facilitators are in the residency clinics 4 days per week, with 1 day spent in department offices to meet and discuss projects at the Hub meeting 
Figure 2. Department of Family Medicine and Community Health (DFMCH) Faculty Publications, 2015-2020 *Family Physicians Inquiries Network (FPIN) HelpDesk Answers publications.

12

\section{Average Scholarly Products}

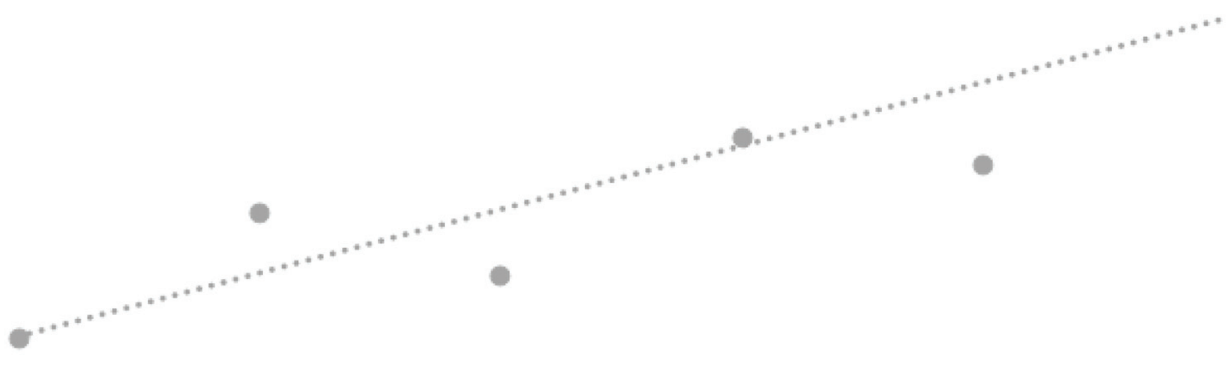

Department Goal for Clinical Faculty: 1 article / year

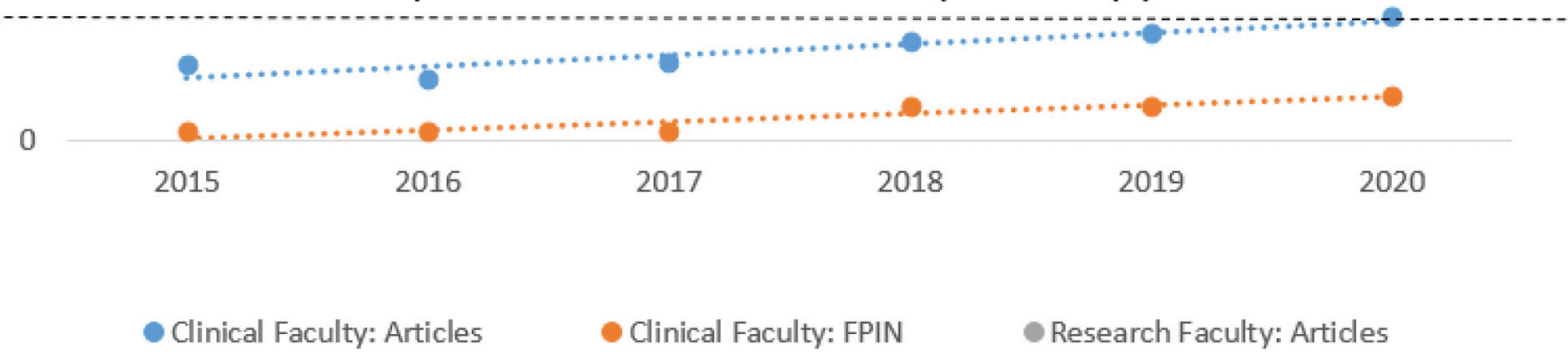

(described below). Research facilitators' roles include the following: assisting with regulatory needs and application development (eg, Institutional Review Board applications, progress reports, grant applications), formulating research questions, study design, implementation of projects, coordinating research and scholarship meetings at each clinic, facilitating research and scholarship projects across faculty and clinics, participating in CSI, and assisting in the dissemination of project results, such as manuscript preparation and poster or conference presentation materials.

2. Other research staff and managers were added to the research services infrastructure to facilitate faculty research and scholarship, including a database manager (sixth author), data entry/survey development staff, a statistical analyst, and a 
computer programmer. A final addition covered $10 \%$ to $20 \%$ effort of 2 career research faculty with expertise in quantitative and qualitative research to consult with clinical faculty on their research and scholarship projects.

A weekly Hub meeting was established to discuss research and scholarship activities happening in the residency clinics and at the larger department level. These meetings serve to keep research staff informed about departmental research and scholarship activities, promote collaborations across faculty, clinics, and departments, and provide training and resources to advance state-of-the-science research and scholarship activities in the DFMCH.

\section{Online "Portal"}

As clinical faculty involvement in research and scholarship, activities increased, and the Hub provided more resources to assist faculty, there was a need for a robust system to coordinate and communicate the research and scholarship activities in the DFMCH. In response, an online portal was developed by our computer programmer that allowed for seamless communication, efficiency in resource delivery and consultation services, and ease of fostering collaborations across clinics and faculty. The portal design also serves as an educational tool for clinical faculty and residents by asking questions to prompt them to ensure project readiness, rigor, and success. At the same time, the portal was also intended to keep research and scholarship activities at the forefront in each of the residency clinics. For example, the research proposed in the portal gets vetted first at the clinic level to ensure it aligns with the clinic's goals, will not burden the clinic resources, and has enough support to be successfully conducted in the clinic. Once approved by the clinic, the proposal is routed to the Hub, and additional resources, consultations, or services required are approved and conducted via the Hub.

\section{Clinic-Level Research Review Committee}

A clinic research review committee was formed in each of the 4 UMN clinic residency programs. The main goal of these committees is to keep research front-and-center in the clinics. These committees meet monthly to discuss the research projects proposed using the process described above. This committee also reinforces buy-in and support at the clinic level for research and scholarship activities.

\section{Discovery Seed Grant Awards}

To promote and incentivize research and scholarship activity among faculty, Discovery Seed Grant Awards were created using philanthropy funding. These $\$ 5000$ grants are awarded to 2 departmental faculty annually and disbursed over 24 months to provide resources for novel research projects to launch and gain momentum.

\section{Initial Outputs and Outcomes of the DFMCH Transformation to Date}

During our harmonized transformation, we tracked outputs and outcomes. Several outputs of our efforts have already been described above (eg, CSI, the Hub, online Portal, Discovery Seed Grant Awards).

Key outcome metrics were tracked to identify the initial impact of our transformation process, including peer-reviewed publications, grants submitted and awarded, and research training. To ensure that published articles were high quality, we used the following criteria to select publications: (1) peer-reviewed and (2) indexed in Scopus. Analyses were then performed using SPSS version 26 Curve Estimation. For each year, from 2015 to 2020, the average number of publications, grant submissions, and grants awarded per faculty member were calculated. This was done separately for clinical and research faculty. Rates of grants awarded per year were calculated by dividing the number of grants awarded each year by the number of applications submitted. This again was done individually for clinical and research faculty. In addition, research training (ie, advanced research degrees, CSI graduates, percentage faculty with research training) was also tracked across the 5 years. Each of the described variables was plotted across years, and a linear trend line was fit. Changes in variables across time are indicated by the slope of the trend line, which is indicated by the $\beta$ coefficient and tested for difference from 0 using the $t$ statistic.

In Figure 2, results show a statistically significant increase each year in the number of publications by clinical faculty, both journal articles $(\beta=0.10$; $\left.\mathrm{R}^{2}=0.75 ; \quad P=.026\right)$ and peer-reviewed FPIN's $\left(\beta=0.07 ; \mathrm{R}^{2}=0.88 ; P=.005\right)$, and a statistically significant increase in career research faculty publications $\left(\beta=0.52 ; \mathrm{R}^{2}=0.78 ; P=.02\right)$. In addition, the departmental goal for faculty publications was met or exceeded by both clinical faculty (ie, 1 
publication per year expected) and research faculty (ie, 3 publications per year expected) by the year 2020.

In Table 1, faculty grants submitted and awarded are shown. Findings show that clinical faculty on average submitted 0.10 grants per year $(\beta=-0.002$; $P>.05)$ and research faculty submitted on average 3 grants per year $(\beta=0.24 ; P>.05)$, which did not significantly increase or decrease over the 5 -year transformation. This finding is not unexpected because once faculty receive grant funding, they often do not submit new grants until their awards are in the last few years of funding. In addition, we did not intentionally target grant funding as part of our transformation process (eg, CSI focused on manuscript writing); instead, manuscripts were the priority. Thus, we did not necessarily expect to see a change in this measure. Importantly, however, grant funding stayed consistent and did not significantly reduce during the harmonized transformation period for clinical or research faculty. Furthermore, according to the Blue Ridge rankings, our goal to be in the top 3 most-NIH-funded family medicine departments was accomplished, and we have stayed at \#3 for the last 3 years. ${ }^{26}$

In Table 2, the number of faculty with advanced research training, the number of CSI graduates, and the percent of faculty trained in how to do research are shown. Findings indicate statistically significant increases in the number of faculty with advanced research training $\left(\beta=1.40, \mathrm{R}^{2}=0.98\right.$, $P=.01)$; the number of CSI graduates $(\beta=6.10$, $\left.\mathrm{R}^{2}=0.97, P=.018\right)$, and increases in faculty trained to engage in research $\left(\beta=0.09, \mathrm{R}^{2}=0.97, P=.016\right)$ over the 5-year transformation process. This increase in research and scholarship knowledge and skills is particularly meaningful because it addresses 1 key barrier that clinical faculty often mention of not feeling adequately trained to engage in research and scholarship activities.

\section{Lessons Learned in the DFMCH Harmonized Transformation}

There were several lessons learned in our transformation that fall under 6 larger themes.

Lesson Learned \#1: Key Elements of the Transformation Need to Be Balanced as an Ensemble

Harmonizing our mission across clinic, education, and research was a critical aspect of our transformation. 
Table 2. Department of Family Medicine and Community Health (DFMCH) Faculty Research Training by Year

\begin{tabular}{lccc}
\hline & $\begin{array}{c}\text { Advanced Research Training } \\
(\text { Masters or PhD) }\end{array}$ & $\begin{array}{c}\text { Percentage of Clinical Faculty } \\
\text { with Research Training }\end{array}$ \\
\hline 2014 & 18 & NA & $27 \%$ \\
2015 & 20 & NA & $29 \%$ \\
2016 (15 CSI course finished in & 21 & 7 & $41 \%$ \\
Feb 2016) & 22 & 15 & $50 \%$ \\
2017 & 24 & 22 & $62 \%$ \\
2018 & 25 & 25 & $66 \%$ \\
2019 & 27 & 35 & $73 \%$ \\
2020 & & & \\
\hline
\end{tabular}

CSI, Collaborative Scholarship Initiative.

This allowed for research to be a part of every conversation occurring across the department. Thus, research and scholarship were front-and-center when all decisions were being made related to clinical and educational goals and vice versa. In addition, while not a linear process, it was important that the elements of our harmonized transformation were implemented simultaneously across our 5-year transformation to increase the likelihood of sustainability. For example, creating resources for faculty, such as manuscript writing groups without providing faculty protected research and scholarship time, or without the addition of research facilitators to coordinate the process, might result in a project being conceived, but stalling out, being undertaken less rigorously, or never coming to fruition.

Lesson Learned \#2: Cultural and Organizational Shifts Take Concerted Effort and Time

It is possible to create a culture of research and scholarship within a department of family medicine. It requires not only consistent messaging and support from central leadership over time but also intentional action and fiscal backing for infrastructure and activities necessary for growth. For example, our harmonized transformation to build capacity for all-faculty research and scholarship transformation took over 5 years to carry out, and we continue to improve protocols and systems to ensure sustainability.

Lesson Learned \#3: Embrace Iteration: Allow "Bumps in the Road" to Propel the Work Forward One example of a "bump in the road" was right sizing our research facilitators. Early in our transformation, we decided to provide a dedicated research facilitator for each residency clinic, but it was unclear how much time was needed. Over the 5 years of harmonized transformation, research facilitators' efforts ranged from $30 \%$ to $100 \%$, with $75 \%$ to $100 \%$ determined ideal. Research facilitators started out being in the clinics 1 day a week, but this grew to 4 days. We found it important to integrate the research facilitator into the clinic to understand the clinic processes/flow better, build relationships with clinic staff and faculty, and be available to meet when faculty have administrative time. In the first year of this work, we had a high percentage of projects that did not come to fruition (eg, study design issues, feasibility of project in a busy clinic setting)_having the research facilitator regularly involved and the other resources available in the Hub, the quality and success of projects have increased.

\section{Lesson Learned \#4: Transformation is Financially Feasible}

Our transformation could not have been accomplished or sustained without a financially feasible approach. Several important financial decisions were made. First, we identified what types of support and resources were needed to increase faculty research and scholarship output. Second, we reorganized some roles and redeployed current staff to support this initiative. For example, our research facilitators existed in our department already as either prior project directors for our career research faculty or other departmental tasks but were redeployed as research facilitators in our new model. While the majority of research facilitator time is departmental funded, they are also supported on grant funding. Third, some efforts (eg, Discovery Seed Grants) are funded by philanthropy. 
Lesson Learned \#5: Career Research Faculty Can Mutually Benefit from Clinical Faculty Engaging in Scholarship

The harmonization transformation benefitted not only our clinical faculty but also our career researchers. As can be seen from Figure 2, career research faculty also increased their publications. In addition, career research faculty's grant funding has maintained our position in the top 3 of most NIH-funded researchers according to the Blue Ridge rankings. ${ }^{26}$ These successes are likely due to the new collaborations with our clinical faculty, more robust resources available, and the support focused on research and scholarship in the department. The career research faculty also help teach the CSI course. Their participation is covered through their departmental allocation of service time. They typically participate in 1 to 2 CSI sessions per year; thus, this is not a high level of expectation of involvement but enough to become familiar with the clinical faculty and form potential collaborations.

Lesson Learned \#G: Honor Skepticism or Disinterest and Let People Cultivate Enthusiasm for Research and Scholarship Activities Rather than Being Forced

Not every clinical faculty member sees themselves as a researcher or imagined that this role would (or could) be part of their job. In addition, clinic managers or staff may not initially think that research and scholarship activities are a good fit for clinical contexts (eg, burden to clinic workflow, additional meetings). During the harmonized transformation, it was critical first to listen and be responsive to concerns and then work to find compromises and develop solutions without being experienced as coercive to skeptical individuals. In addition, it was useful to highlight innovative and useful research and scholarship activities occurring in other clinics to exemplify that research and scholarship activities are possible, beneficial, and even an enjoyable addition to clinical and educational missions. Furthermore, providing incentives, such as the Discovery Seed Grant Award or supported time away from clinic such as our CSI course, was experienced by our faculty as an investment in them, and many got excited about research and scholarship opportunities even when at first, they did not seem interested.

There are important limitations of our work to acknowledge that are also opportunities for future research. First, measurement of collaboration growth across faculty on publications, presentations, and funding opportunities would be important to capture in future research. In addition, measures such as resident scholarship that allow for measuring whether the services created for faculty benefit others more broadly would be important to obtain and measures of faculty, resident, and staff satisfaction with the research services hub. Furthermore, an economic analysis showing the impact of faculty's protected time (ie, $10 \%$ for scholarship and research) on scholarship and research productivity would be helpful to collect in the future, in addition to the overall costs of the research services hub.

\section{Conclusion}

The DFMCH at the UMN engaged in a 5-year transformation to expand research and scholarship opportunities to all faculty. Harmonization across the 3 missions of clinic, education, and research was used. This model of harmonized transformation may be useful for other family medicine departments or other academic programs.

To see this article online, please go to: http://jabfm.org/content/ 34/5/1055.full.

\section{References}

1. Martin JC, Avant RF, Bowman MA, et al. The future of family medicine: a collaborative project of the family medicine community. Ann Fam Med 2004; Suppl1:S3-32.

2. Zerhouni EA. Translational and clinical science Time for a new vision. N Engl J Med 2005;353: 1621-3.

3. Scherger JE, Young HF. The AAFP research initiative. J Fam Pr 1998;46:203-4.

4. Bowman M, Lucan S, Rosenthal T, Mainous AI, James P. Family medicine research in the United States: from the late 1960's into the future. Fam Med 2018;49:289-95.

5. Ewigman B, Davis A, Vansaghi T, et al. Building research \& scholarship capacity in departments of family medicine: A new joint ADFM-NAPCRG initiative. Ann Fam Med 2016;14:82-3.

6. deGruy FV, Ewigman B, DeVoe JE, et al. A plan for useful and timely family medicine and primary care research. Fam Med 2015;47:636-42.

7. Hester CM, Jiang V, Bartlett-Esquilant G, et al. Supporting family medicine research capacity: the critical role and current contributions of US family medicine organizations. Fam Med 2019;51:120-8.

8. Gotler RS. Unfinished business: the role of research in family medicine. Ann Fam Med 2019;17:70-6. 
9. Peek CJ. The 5R's: An emerging bold standard for conducting relevant research in a changing world. Fam Med 2019;51:103-11.

10. Liaw $W$, Petterson $S$, Jiang $V$, et al. The scholarly output of faculty in family medicine departments. Fam Med 2019;51:103-11.

11. Eder M, Pierce J. Innovations in faculty development: study of a research assistance unit designed to assist clinician-educators with research. South Med J 2011;104:647-50.

12. Kempainen RR, Mckone EF, Rubenfeld GD, Scott CS, Tonelli MR. Publications and extramural activities of general internal medicine and medicine subspecialty clinician - educators: a multicenter study. 2005;80:238-43.

13. Levinson $W$, Rubenstein A. Integrating clinicianeducators into academic medical centers: challenges and potential solutions. Acad Med 2000;75:906-12.

14. Peek CJ, Windenburg D. Harmonized Transformation Across Care, Education, and Research: What does it really mean and is it possible? In: Workshop at 45th North American Primary Care Research Group (NAPCRG) Annual Meeting. Available from: https://www.napcrg. org/conferences/annualpastmeetingarchives/. Published 2017. Accessed July 15, 2021.

15. Ranwala D, Alberg AJ, Brady KT, Obeid JS, Davis R, Halushka PV. Scientific retreats with 'speed dating': networking to stimulate new interdisciplinary translational research collaborations and team science. J Investig Med 2017;65:382-90.

16. Brandon C, Jamadar D, Girish G, Dong Q, Morag Y, Mullan P. Peer support of a faculty "writers' circle" increases confidence and productivity in generating scholarship. Acad Radiol 2015;22:534-8.

17. Skarupski KA, Foucher KC. Writing Accountability Groups (WAGs): a tool to help junior faculty members build sustainable writing habits. J Fac Dev 2018;32:47-54.

18. Hekelman FP, Gilchrist V, Zyzanski SJ, Glover P, Olness K. An educational intervention to increase faculty publication productivity. Fam Med 1995; 27:255-9.

19. Roth LM, Neale AV, Kennedy K, Dehaven MJ. Insights from practice-based researchers to develop family medicine faculty as scholars. Fac Dev 2007;39:504-9.

20. Buffington ALH, Lange C, Bakker C, et al. The Collaborative Scholarship Intensive: A research-intensive course to improve faculty scholarship. Fam Med. 2021; May;53(5):355-58.

21. Liaw $W$, Eden A, Coffman $M$, Nagaraj $M$, Bazemore AW. Factors associated with successful research departments: a qualitative analysis of family medicine research bright spots. Fam Med 2019;51:87-102.

22. Peek CJ. Mission and vision for the department of family medicine and community health, University of Minnesota Medical School. Intern Rep. 2012. Unpublished.

23. Xierali IM, Nivet MA, Syed ZA, Shakil A, Schneider FD. Trends in tenure status in academic family medicine, 1977-2017: implications for recruitment, retention, and the academic mission. Acad Med 2020; 95:241-7.

24. Johnston SC. Academic medical centers: too large for their own health? JAMA 2019;322:203-4.

25. Boyer EL. Scholarship Reconsidered: Priorities of the Professoriate. 1990. Josey-Bass, San Francisco, CA.

26. Blue Ridge Institute for Medical Research. Available from: http://www.brimr.org/. Published February 9, 2021. Accessed April 23, 2021. 